\title{
Transcriptional markers in response to hydrocortisone in sepsis in ADRENAL: a step toward precision medicine
}

Timothy E. Sweeney ${ }^{1^{*}}$ (D) and Hector R. Wong ${ }^{2,3}$

๑ 2021 Springer-Verlag GmbH Germany, part of Springer Nature

Practice varies widely for corticosteroid usage in sepsis [1], perhaps because study results have themselves varied widely, leading to a minor recommendation within the SSC guidelines for patients in refractory shock. Still, a recent Cochrane review of 61 trials with over 12,000 participants suggests a modest but significant benefit in terms of 28-day mortality along with reduced ICU and hospital length of stay [2]. So why does practice continue to vary? Individual study results vary; the climax of this scientific dilemma occurred 2018 when two independent large RCT were published in the same issue of NEJM with contradictory results (APROCCHSS and ADRENAL; the studies also had differences in severity of illness and steroid dosing). The largest study of these, ADRENAL $(N=3658)$, showed no mortality benefit with corticosteroids, but shorter time in shock and on mechanical ventilation [3]. In addition, there are negative side effects; the same Cochrane review showed increased rates of hypernatremia and hyperglycemia with corticosteroid usage.

Sepsis is a syndrome, not a well-defined disease, and as a result encompasses broad heterogeneity. Rather than all patients experiencing a small consistent benefit from steroids, it is assumed (but not assured [4]) that modest results at the population level are due to some patients with sepsis experiencing strong benefit, while others are not affected or are harmed (a heterogeneity of treatment effect). This matches clinical practice; many intensivists have experienced a patient make a seemingly dramatic recovery when given corticosteroids, yet others continue

\footnotetext{
${ }^{*}$ Correspondence: tsweeney@inflammatix.com

${ }^{1}$ Inflammatix, Inc., Burlingame, CA 94010, USA

Full author information is available at the end of the article
}

to decline. Pre-identifying which septic patients will benefit from steroids is thus an obvious next step.

In a prespecified subgroup analysis of the ADRENAL trial $(N=494)$, Cohen et al. attempt to determine whether such a steroid-responsive subgroup could be identified based on circulating whole blood gene expression of a narrow group of corticosteroid-response-related mRNAs [5]. The study has several strengths; it follows a prespecificed protocol of a rigorous blinded RCT, and the limited number of biologically plausible genes and outcomes is statistically rigorous and lowers the chance of false-positive findings. The authors found no differences in mortality according to gene expression (either alone or in association with steroids), but did show a significant interaction between gene expression and time to shock reversal for two genes, GLCCI1 (HR 3.81 vs. 0.64) and HSD11B1/BHSD1 (HR 0.55 vs 1.32) in hydrocortisonetreated vs. placebo groups, respectively. While not as 'desirable' a goal as improving survival, a low-cost, rapid test (e.g. qRT-PCR or qRT-LAMP) that could identify a subgroup with faster shock resolution with steroids could improve outcomes and lower costs (Fig. 1).

That said, an important note is that rather than use qRT-PCR to study their genes of interest, the authors ran RNAseq on all samples, a non-standard approach for targeted gene expression studies. While this does not cast doubt on the overall findings, it does require some sensitivity analysis of the overall bioinformatics analysis used, which generally shows the findings hold up well regardless of methods of normalization. Furthermore, the approach has generated a rather large gene expression data set, which opens the possibility for further analyses. In other words, while the authors have shown a significant effect of 2 of 11 genes studied, they have not studied 


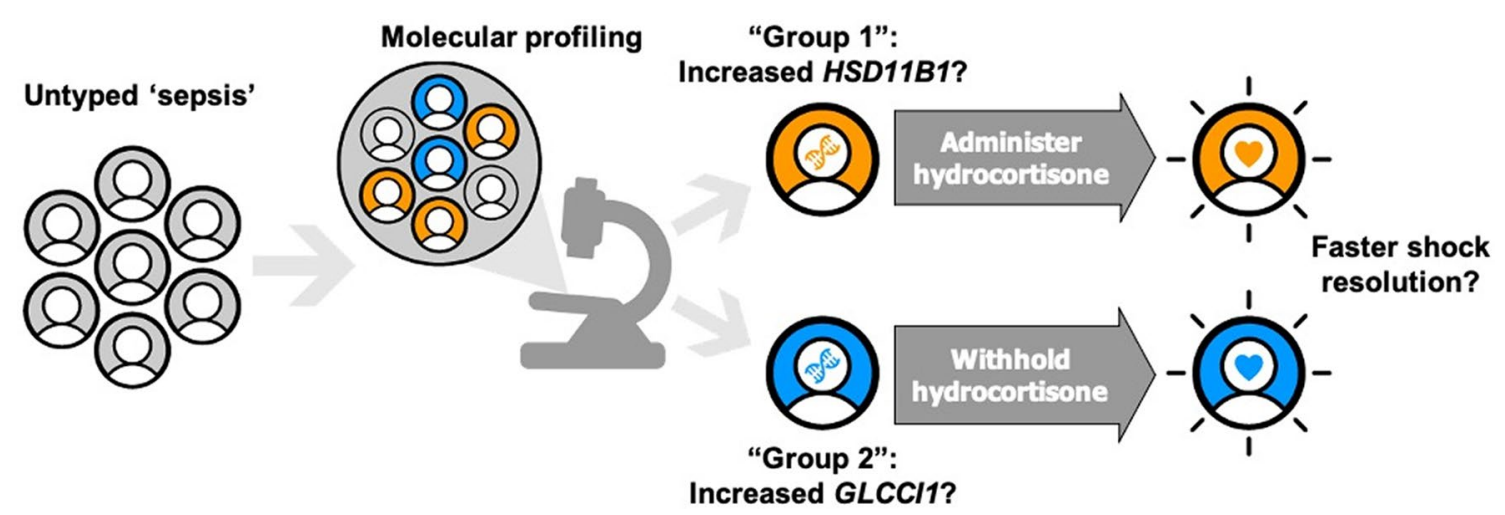

Fig. 1 Molecular profiling of sepsis patients to determine hydrocortisone sensitivity. Different molecular markers may indicate either a group of patients that benefits from steroid treatment, or a group likely to experience harm. Here, HSD11B1 and GLCCI1 are suggested, though further study is needed to determine the optimal molecular profiling test

whether there are substantially more effective ways to identify a subgroup that would benefit from steroids (ideally with a significant effect on mortality). They also have elected not to publish data beyond the 11 genes, which we assume means they are performing other analyses on the broader data set.

Further studies on these (or similar) data could entail, for instance: (1) a broader search across the hundreds of possibly steroid-response-related genes for a greater effect size (though of course, multiple hypothesis testing comes into account), or (2) an application of previously defined transcriptomic endotypes, at least three of which have shown potential effectiveness in identifying steroid-responsive subgroups [6-8], or (3) training of novel machine-learning algorithms using the whole transcriptome for prospective validation in prospective, algorithm-enriched RCTs. Further experiments with slightly different data could entail longitudinal sampling (perhaps the trajectory of gene expression or gene expression after initial steroid use could inform better which patients would benefit) or could pair transcriptomic data with DNA analysis for SNPs to determine whether differential gene expression is happening in the acute phase or is representative of an eQTL driving steroid responsiveness. Overall, a broader analysis of the transcriptomic data is more likely to allow for identifying biological subgroups linked to steroid treatment outcomes.

Another kind of follow-up of course would be to repeat these findings using external data. We looked in publicly available transcriptomes from CORTICUS [9] and VANISH [10] for the 11 genes studied by Cohen et al., and were able to find data for all but GR_BETA and LINC02210-CRHR1. We found no significant interaction for any gene with hydrocortisone treatment in mortality outcomes in CORTICUS, matching at least that finding of Cohen et al. We did find a significant association of $\mathrm{NR} 3 \mathrm{C} 2$, but none of the other genes, with mortality in hydrocortisone treatment in VANISH. However, these cohorts are small, and we suggest that our findings show the variable nature of single-gene associations and emphasize the need for further, larger studies. Unfortunately, neither cohort had shock resolution as a publicly available outcome measure.

The positive findings linking GLCCI1 and HSD11B1 expression to shock reversal upon hydrocortisone treatment suggest several important future directions. First, they are further evidence that transcriptomics may allow identification of steroid-responsive subgroups in sepsis. Every future trial of corticosteroids should at least be gathering RNA-preserved blood (e.g., in PAXgene RNA tubes) to allow for similar post-hoc analysis, and such trials should report standardized data on both short-term and long-term outcomes, so that study findings can be pooled. Second, these present findings need to be further replicated across different clinical settings, including in particular a broad range of demographics and clinical features (e.g., site of infection), to better understand the generalizability of the signal. Third, prior to clinical practice, a rapid diagnostic test needs to be made and validated, but it is unlikely that test development will begin without a more thorough study of further genes or endotypes to look for a higher effect size. Such an effort would require rigorous development of an analytically valid assay (probably using RT-PCR or similar). Finally, of course, a prospective, randomized interventional study showing improved outcomes based on the validated rapid test would be necessary prior to implementation in clinical practice. Still, the conclusion is clear: the era of precision medicine in sepsis, matching the right therapies to the right patients, is drawing nearer. 


\section{Author details}

1 Inflammatix, Inc., Burlingame, CA 94010, USA. ${ }^{2}$ Division of Critical Care Medicine, Cincinnati Children's Hospital Medical Center and Cincinnati Children's Research Foundation, Cincinnati, OH 45223, USA. ${ }^{3}$ Department of Pediatrics, University of Cincinnati College of Medicine, Cincinnati, OH 45267, USA.

\section{Acknowledgements}

We thank Chris Wilson for graphic design and Florian Uhle for helpful commentary.

\section{Declarations}

\section{Conflicts of interest}

Dr. Sweeney is an employee of, and stockholder in, Inflammatix, Inc, which is working on sepsis endotyping tests. Dr. Wong and the Cincinnati Children's Hospital Research Foundation hold U.S. patents for endotyping strategies for sepsis.

\section{Publisher's Note}

Springer Nature remains neutral with regard to jurisdictional claims in published maps and institutional affiliations.

Received: 30 June 2021 Accepted: 5 August 2021

Published online: 10 August 2021

\section{References}

1. Bruno JJ, Dee BM, Anderegg BA, Hernandez M, Pravinkumar SE (2012) US practitioner opinions and prescribing practices regarding corticosteroid therapy for severe sepsis and septic shock. J Crit Care 27(4):351-361. https://doi.org/10.1016/j.jcrc.2011.12.011
2. Annane D, Bellissant E, Bollaert PE et al (2019) Corticosteroids for treating sepsis in children and adults. Cochrane Database Syst Rev. https://doi. org/10.1002/14651858.CD002243.pub4

3. Venkatesh B, Finfer S, Cohen J et al (2018) Adjunctive glucocorticoid therapy in patients with septic shock. N Engl J Med 378(9):797-808. https://doi.org/10.1056/NEJMoa1705835

4. Senn S (2004) Individual response to treatment: is it a valid assumption? BMJ 329(7472):966-968. https://doi.org/10.1136/bmj.329.7472.966

5. Cohen J, Blumenthal A, Cuellar-Partida G et al (2021) The relationship between adrenocortical candidate gene expression and clinical response to hydrocortisone in patients with septic shock. Intensive Care Med. https://doi.org/10.1007/s00134-021-06464-5

6. Wong HR, Cvijanovich NZ, Anas N et al (2015) Developing a clinically feasible personalized medicine approach to pediatric septic shock. Am J Respir Crit Care Med 191(3):309-315. https://doi.org/10.1164/rccm. 201410-18640C

7. Antcliffe DB, Burnham KL, Al-Beidh F et al (2019) Transcriptomic signatures in sepsis and a differential response to steroids from the VANISH randomized trial. Am J Respir Crit Care Med 199(8):980-986. https://doi. org/10.1164/rccm.201807-14190C

8. Iglesias J, Vassallo AV, Liesenfeld O et al (2020) A 33-mRNA classifier is able to produce inflammopathic, adaptive, and coagulopathic endotypes with prognostic significance: the outcomes of metabolic resuscitation using ascorbic acid, thiamine, and glucocorticoids in the early treatment of sepsis (ORANGES) trial. J Pers Med. https://doi.org/10.3390/jpm11 010009

9. Sprung CL, Annane D, Keh D et al (2008) Hydrocortisone therapy for patients with septic shock. N Engl J Med 358(2):111-124. https://doi.org/ 10.1056/NEJMoa071366

10. Gordon AC, Mason AJ, Thirunavukkarasu N et al (2016) Effect of early vasopressin vs norepinephrine on kidney failure in patients with septic shock: the VANISH randomized clinical trial. JAMA 316(5):509-518. https:// doi.org/10.1001/jama.2016.10485 